\title{
Mathematical Modeling to Inform Policy
}

\author{
Jaline Gerardin \\ Northwestern University, USA
}

\begin{abstract}
In 2020, the US state of Illinois assembled a modeling task force to help inform COVID-19 policy. We show how local modelers built transmission models to capture local trends and make short-term forecasts. We take a step back and ask where the state could have done better in preventing disparities and whether we could have known that we were underprepared for reopening. We describe a sentinel surveillance scheme for early warning of increasing trends and show what worked and what didn't work in implementing this surveillance. Finally, we present some thoughts on lessons learned in engaging with public health officials during the pandemic.
\end{abstract}

